\section{Abstractions}

\section{FIRST AUTHOR}

For Robin Canup, studying the celestial pas de deux of planet and satellite formation is linked to more Earthbound dances. Until six years ago, Canup, who is director of the Southwest Research Institute's Department of Space Studies in Boulder, Colorado, was prima ballerina with the Boulder Ballet. Together with co-author William Ward, she combined an artistic mindset with scientific endeavour to try to understand why the Solar System's gaseous planets and their satellite systems have a nearly constant mass ratio. The results, published on page 834 , provide clues on the choreography of planet formation. Canup spoke to Nature about her work - and the link between space science and dance.

What's the relationship between ballet and studying planet and satellite formation? In many creative endeavours, you have very structured and formal techniques that are the foundations upon which you try to do new things. In ballet, that structure is the classical technique. In space science, it is physics and mathematics. In both science and art, it is important to distil a problem down to its fundamentals as much as possible, while still expressing yourself creatively. The formal rules seem constraining in some respects. But ultimately they are liberating because they provide you with time-tested forms within which to work.

What is the significance of the mass ratio? We believe the satellites formed in the presence of gas - mainly hydrogen - because the planet was in the end stages of formation. As the planet swept in gas and solid material, a disc formed around it, and the satellites formed within this disc. The gravity of a satellite induces spiral waves in the gas disc, and gravitational interactions between these waves and the satellite cause the satellite's orbit to contract. This effect becomes stronger as a satellite grows, so that the bigger a satellite gets, the faster its orbit decays, implying a maximum satellite size.

\section{How did you model this system?}

We developed a numerical simulation that tracks the inflow of solids and gas into orbit around the planet, and followed the growth of satellites, their orbital evolution and their loss due to collision with the planet.

How does your dance background extend to your scientific management style? Scientists are a lot like artists; each person has their own unique style. So to whatever extent possible, I try to give people the freedom to work in the manner that is best for them. You want to try to create an environment that encourages talented people to do their best creative work.

\section{MAKING THE PAPER}

Jesús Mavárez

\section{Hybrids - and a marker pen - offer insights into a butterfly family tree.}

The various species of Heliconius butterfly found across Central and South America have long intrigued evolutionary biologists. Between them, the 60 or so species display a dazzling array of specific colour patterns - a diversity that is very hard to explain by genetic mutation alone. This led to the suggestion that some of the species arose as hybrids.

On page 868, Jesús Mavárez and his colleagues present evidence confirming that Heliconius heurippa is a hybrid of $H$. cydno and $H$. melpomene. They also show that $H$. heurippa uses wing markings as a guide to select other hybrids as mates and so propagate its species. All three butterflies have black wings, but $H$. melpomene sports red markings, $H$. cydno has yellow markings, and the hybrid $\mathrm{H}$. heurippa features both red and yellow marks.

"We decided to attack the problem using three different strategies. That is what made our study so strong," says Mavárez, who is a postdoc at the Smithsonian Tropical Research Institute in Panama. For the first part, the researchers sought out genetic markers that would allow them to distinguish between $H$. cydno and H. melpomene - no mean feat as the butterflies' genomes are almost identi$\mathrm{cal}$. Further analysis revealed that $H$. heurippa shares some genetic markers with the other two species, as well as having some unique markers of its own. This strongly suggests that H. heurippa is indeed a hybrid.

The team next put this prediction to the test by exploiting the butterfly-handling expertise of their collaborators at the University of the Andes in Bogota, Colombia. By crossing H. melpomene and H. cydno, they recreated H. heurippa in the lab. "We thought it would be hard to do, but in three generations of crosses we were able to create a phenotype indistinguishable from wild-type H. heurippa," says Mavárez.

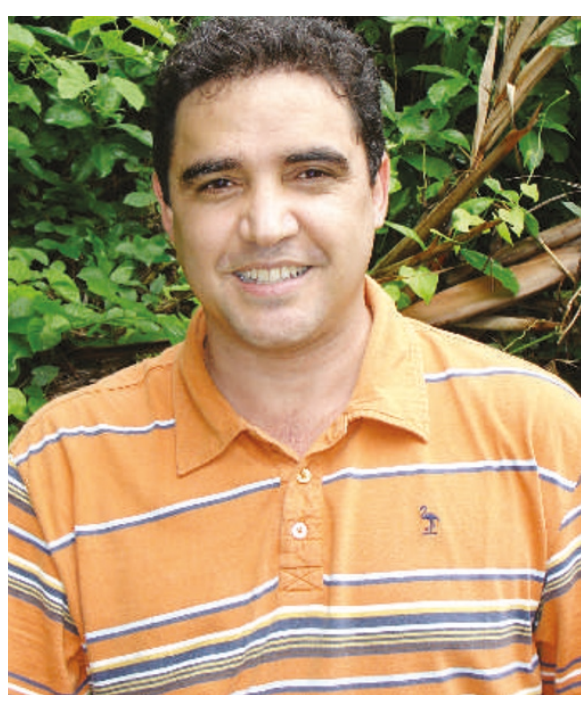

But the team still needed to show that these hybrids could survive and propagate. If the hybrids chose either of the parental species as mates in preference to other hybrids, the new species would be doomed. So the researchers exposed $H$. heurippa males to females from all three species. Invariably, the males went for mates of their own kind. When the scientists modified the wing patterns of $H$. heurippa females - by 'erasing' either the yellow or red bands using a black marker - the males snubbed them.

"Somehow when these butterflies inherited both colour patterns, they also inherited the preference for both markings," says Mavárez, who now plans to investigate the genetics behind this behaviour.

The trickiest part of the experiment, says Mavárez, was maintaining the stock of butterflies long enough to do the experiments. Two students made this part of the project possible with very few resources, says Mavárez. Gathering the butterflies was also a challenging and sometimes dangerous task. "We were lucky that $H$. heurippa and H. melpomene are found near Bogota, which is a relatively safe area of Colombia," he says. But H. cydno comes from a more dangerous part of the country, so the team had to go to Venezuela to get specimens.

\title{
ON THE WEB OPEN PEER-REVIEW DEBATE
}

Five new contributions to Nature's online debate about open peer review are published today.

\section{Systems: An open, two-stage peer-review journal \\ Thomas Koop and Ulrich Pöschl}

Technical solutions: Wisdom of the crowds

Chris Anderson

Quality and value: Statistics in peer review

David Ozonoff
Ethics: What is it for?

Elizabeth Wager

Perspective: The pros and cons of open peer review

Thomas DeCoursey

All articles are available at: www.nature.com/nature/peerreview/debate/ index.html

Readers are invited to join the debate in Nature's peer-review comments blog at: http://blogs.nature.com/nature/peerreview/ debate/comments 